\title{
Carbapenem-Resistant Enterobacteriaceae Co-Infections with Klebsiella; a Retrospective Study
}

\section{Abstract}

Background: Carbapenem antibiotics are often used to treat bacterial infections that are refractory to other more commonly used antibiotics. The resilience of bacteria and their resistance mechanisms are of novel interest in the clinical setting, as antibiotic resistant bacteria are posing a challenge to health care professionals. The goal of this retrospective chart review was to isolate Carbapenem resistance among co-infections of Klebsiella and other microorganisms and to conduct a systematic review of this antibiotic resistance.

Methods: This was a retrospective, single center chart review of patients admitted to Kingsbrook Jewish Medical Center from June 2011 to June 2014.

Study design: Retrospective Study, Chart Review.

Cases: Two patients met the criteria of the investigators and their charts were reviewed.

Results: Two cases with Klebsiella and E. coli co-infection with Carbapenem-resistant strains were identified. Patient 1 had a coinfection of Klebsiella and E. coli. Initially, Klebsiella was carbapenem resistant, while $E$. coli was carbapenem susceptible. However, eventually both bacterial strains developed resistance. Patient 2 had a co-infection of Klebsiella and E. coli which were both carbapenem resistant. However, in contrast to Patient 1 , Klebsiella became susceptible to carbapenem.

Conclusion: The findings indicate that carbapenem-resistant Klebsiella can be transient. Investigation is warranted to explore influence genetic traits, iatrogenic association and environmental causality of CRR.
Richard A. Giovane ${ }^{1}$, Steven Brooks ${ }^{2}$

1 From St. George's University School of Medicine, Grenada West Indies.

2 Clinical Assistant Professor of Pharmacology, Department of Infectious Disease Kingsbrook Jewish Medical Center, 585 Schenectady Ave, Brooklyn, New York, 11203.

\section{Contact information:}

Richard A. Giovane BS.

”rgiovane@sgu.edu

Keywords

Carbapenems; Carbapenemresistant; Enterobacteriaceae co-infections; Klebsiella; Enterobacteriaceae ; KPC-2; KPC-3; OmpF; OmpC; marRAB 


\section{Introduction}

The use of carbapenem antibiotics such as imipenem and meropenem has increased significantly in the past several years. This has been due to increased antibiotic resistance of many species of bacteria in the hospital setting associated with acquisition of one or more resistance mechanisms. Carbapenem antibiotics are often used to treat bacterial infections that have become refractory to antibiotic treatment attempts using other classes of antibiotics such as cephalosporins and aminoglycosides.

Recently, bacteria such as Klebsiella, E. coli, and $E$ nterobacter have developed resistance to carbapenem antibiotics in the hospital setting. These types of microbes have been designated as carbapenemresistant Enterobacteriaceae (CRE) [1].

CRE is becoming a major problem for hospitalized patients. As speculated by Goren et al., even for healthy individuals, CRE can often outcompete normal gut flora and therefore colonize the gut, which can become a source of infection for patients $[1,2,3]$.

The mechanisms by which Enterobacteriaciae acquire and spread resistance to carbapenem antibiotics have become a point of interest in current research. Mechanisms for transferring or developing resistance include plasmid transfer from species of CRE such as Klebsiella to other microorganisms, decreased porin expression in the outer membrane, and genetic mutations have been proposed as mechanisms of resistance [4].

The focus of this retrospective chart review was on patients who had a co-infection with carbapenem resistant Klebsiella and another microorganism, which ultimately acquired resistance to carbapenem, presumably by a resistance transfer mechanism.

\section{Methods}

This study involved a retrospective, single center chart review of patients that were admitted to Kingsbrook Jewish Medical Center from June 2011 to June 2014, who had positive cultures of both $E$. coli and Klebsiella. Resistance to carbapenam was reviewed on the microbiology report in the patient's chart, and a timeline was constructed from the initial infection to resolution of infection, with special reference to the presence or change in carbapenem resistance. There was no distinction based on site of infection.

\section{Results}

Based on patient chart analysis, two cases were found with Klebsiella and E. coli co-infection with carbapenem-resistant strains. Both patients were admitted from nursing home. Patient 1 had a coinfection of Klebsiella and E. coli in a wound. Initially, Klebsiella was carbapenem resistant, while E. coli was carbapenem susceptible. One week later, when the wound culture was done again, both Klebsiella and $E$. coli were carbapenem resistant. Months after, the patient developed a Klebsiella infection in the urinary catheter that was carbapenem resistant. The patient had co morbidities such as living in a nursing home and having an indwelling catheter.

Patient 2 had an initial urinary tract infection with Klebsiella that was carbapenem resistant. Two weeks later, the patient was found to be co-infected with Klebsiella and E. coli. that was carbapenem resistant. A clean catch urine specimen was conducted two weeks later, and only E. coli was carbapenem resistant while Klebsiella was carbapenem sensitive.

\section{Discussion}

One can speculate that in patient 1, the $E$. coli acquired carbapenem resistance transfered from Klebsiella. Urban et al. demonstrated the presence of E. coli harboring Klebsiella carbapenemase betalactamases as a result of plasmid transfer from Klebsiella to $E$. coli $[5,6]$. In this scenario, Klebsiella would carry the genes titled KPC-2 and KPC-3, both 
of which contribute to Klebsiella being resistant to carbapenem [7]. Interaction between Klebsiella and E. coli would enable plasmid transfer, and would confer the same carbapenem resistance to the $E$. coli.

Another proposed mechanism of E. coli developing carbapenem resistannce would not involve Klebsiella gene transfer, but genetic mutations to the porin on the outer membrane of E. coli. It has been demonstrated that Enterobacteriaceae species, such as $E$. coli, can undergo genetic mutations in genes such as OmpF and $\mathrm{OmpC}$, which are responsible for porin production $[7,8]$. Lack of porin production is associated with resistance to carbapenem, as the drug has no mechanism of crossing the cell membrane and gaining access into the bacteria $[8,9,10]$.

Silencing of the marRAB operon in Enterobacteriaciae species can also be proposed as a means of carbapenem resistance. The mar operon is also linked to porin expression. Studies by Chollet et al. demonstrate that this operon is influenced by the presence of antibotics [11, 12, 13]. The mar operon is regulated by marA and marR, with marA inducing antibiotic resistance and marR, which down regulates antibiotic resistance $[11,12,13]$. MarA and marR are influenced by several factors, one of them being the bacteria's environment. If the bacteria is subjected to antibiotics, marA can be activated to induce resistance, while an environment without antibiotics would enable marR to be activated and down regulate antibiotic resistance $[11,12,13]$. It can be noted that while Klebsiella and E. coli share a similar genomes in regards to the mar operon, it is uncertain if factors from Klebsiella can influence the genome of E. coli [14].

Patient 1 had a long term wound infection that spanned several months. The patient was initially infected May 6, 2011 and continued to be infected until August 22, 2011. It should be noted that in the month of August, the patient's urinary catheter tested positive for Klebsiella that was resistant to carbapenem. One can speculate that long-term infection of the patient's wound would put the patient at a high risk for a secondary infection-in this case a catheter infection. Bisson et al. speculate that an important risk factor for developing an infection with bacteria exhibiting extended antibiotic resistance properties is hospital stay [14]. Moreover, the CDC also supports this notion as the majority of cases of CRE are in patients who are in long-term care facilities [16].

Since the patient was in a nursing home, the individual would be at a greater risk of acquiring a secondary infection. As reported by $C D C$, the rate of CRE in a nursing home is $30 \%$. Lowe et al. speculate that long term care facilities, such as nursing homes, have yet to develop standard protocol for treating patients that have CRE $[17,18]$. Failure to consistently implement standard protocols for treatment and implementation of contact isolation precautions, puts individuals at risk for developing a secondary CRE infection or spreading the CRE infection to other residents.

The medical timeline for patient 2 is presented below (Figure 1). The medical chart showed that there was an initial clean catch urine obtained on 4/5/13 which grew only Carbapenem resistant Kle-

Figure 1.

\section{Patient initially seen 04/05} Clean catch urine obtained

\begin{tabular}{|l|l|l|}
\hline organism & culture & imipenem \\
\hline E.Coli & Negative & N/A \\
\hline Kleb & positive & $R$ \\
\hline
\end{tabular}

04/16 urine specimen

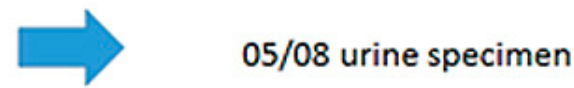

\begin{tabular}{|l|l|l|}
\hline organism & culture & Imipenem \\
\hline E.Coli & Positive & $R$ \\
\hline Kleb & positive & $R$ \\
\hline
\end{tabular}

\begin{tabular}{|l|l|l|}
\hline organism & culture & imipenem \\
\hline E.Coli & Positive & $\mathrm{R}$ \\
\hline Kleb & positive & $\mathrm{S}$ \\
\hline
\end{tabular}


bsiella, A second urine collected on 4/16/13 showed both carbapenem resistant $\mathrm{E}$ coli and Klebsiella. On 5/8/13 a third urine culture grew carbapenem resistant E coli and carbapenem susceptible KlebsieIla. Chart review revealed the patient did not have any fever while under hospital care and no elevated WBC

One can only speculate why Klebsiella lost resistance to carbapenem as evidenced by the culture results on 5/8/13. A possible explanation would relate to the marRAB operon and how it is regulated. The marRAB is influenced by the presence of antibiotics [11, 12, 13]. Initially, when the patient had an $E$. coli and Klebsiella infection, the patient was receiving no antibiotic treatment and both species were carbapenem resistant. It can be hypothesized that after several weeks of no antibiotic treatment, there was induction of marR that would silence the marRAB sequence, which would result in increased porins, and therefore, increased susceptibility to carbapenem $[19,20]$. One can speculate as to why Klebsiella lost resistance to imipenem and $E$. coli still retained resistance. The timing of gene regulation can provide an explanation for this. For instance, Klebsiella may induce marR quicker than $E$. coli so it would lose resistance at a faster rate and therefore be more susceptible earlier. The method of gene regulation in bacteria can also provide an explanation to this. Induction of marR is regulated via a cascade. The mechanism of regulation of the marR cascade could be dependent on the bacteria species. Certain bacteria may regulate the gene cascade differently and therefore this would alter gene expression at varying rates.

\section{Conclusion:}

Co-infections with carbapenem resistant strains of Klebsiella and E coli are a point of interest in medical microbiology. The findings suggests that carbapenem-resistant Klebsiella can influence genetic traits (i.e. transfer of carbapenem resistance) of other En- terobacteriaceae such as $E$ coli when present in the same environment or body site.

The phenomenon of carbapenem resistance transfer between different species of bacteria will likely cause more widespread carbapenem resistance and will greatly reduce the usefulness of some of potent antimicrobials

Speculating that Klebsiella can down regulate certain genes can be of interest clinically. Rigorous antibiotic treatment can induce bacteria to up regulate resistance to antibiotics, and therefore, hinder resolution of an infection. Further investigation is needed to identify iatrogenic associations and causality of CRE in order to prevent further bacterial resistance and more importantly, spread of CRE.

\section{Financial Disclosure}

The Authors did not report any potential conflicts of interest.

\section{References}

1. Cdc.gov, (2014). CDC - Carbapenem-resistant Enterobacteriaceae-HAl. [online] Available at: http://www.cdc.gov/hai/ organisms/cre/ [Accessed 2 Jul. 2014].

2. Goren MG, Carmeli Y, Schwaber MJ, Chmelnitsky I, Schechner $\checkmark$, Navon-Venezia S. Transfer of carbapenem-resistant plasmid from Klebsiella pneumoniae ST258 to Escherichia coli in patient. Emerg Infect Dis. 2010; 16(6): 1014-1017. PMID: 20507761

3. Schwaber MJ, Carmeli Y Carbapenem-resistant Enterobacteriaceae: a potential threat. JAMA. 2008;300: 2911-3. PMID: 19109119

4. Livermore DM, Woodford $\mathrm{N}$. The $\mathrm{b}$-lactamase threat in Enterobacteriaceae, Pseudomonas, and Acinetobacter. Trends In Microbiology, 2006; 14: 413-20.

5. 5- Urban C, Bradford PA, Tuckman M, Segal-Maurer S, Wehbeh W, Grenner L, Colon-Urban R, Mariano N, Rahal JJ. 2008. Carbapenem-resistant Escherichia coli harboring Klebsiella pneumoniae carbapenemase $\beta$-lactamases associated with long-term care facilities. Clin. Infect. Dis. 46: e127-e130. PMID: 18419424

6. Iavon-Venezia S, Chmelnitskyl, Leavitt A, Schwaber MJ, Schwartz $D$, Carmeli Y: Plasmid-mediated imipenem-hydrolyzing enzyme KPC-2 among multiple carbapenem-resistant Escherichia coli clones in Israel. Antimicrob Agents Chemother, 2006, 50 (9): 3098-3101. PMID: 16940107 
7. Yoshida T, Qin L, Egger LA, Inouye M. Transcription regulation of ompF and ompC by a single transcription factor, OmpR. J Biol Chem. 2006; 281: 17114-17123. PMID: 16618701

8. Yigit H, Anderson GJ, Biddle JW, Steward CD, Rasheed JK, Valera LL, et al. Carbapenem resistance in a clinical isolate of Enterobacter aerogenes is associated with decreased expression of $\mathrm{OmpF}$ and $\mathrm{OmpC}$ porin analogs. Antimicrob Agents Chemother, 2002. Dec; 46(12): 3817-3822 10.1128/ AAC.46.12.3817-3822.2002. PMID: 12435682

9. Bornet, C., A. Davin-Regli, C. Bosi, J. M. Pages, and C. Bollet. 2000. Imipenem resistance of Enterobacter aerogenes mediated by outer membrane permeability. J. Clin. Microbiol. 38: 10481052. PMID: 10698994.

10. Tzouvelekis, L.S., E. Tzelepi, A.F. Mentis, A.C. Vatopoulos, and A. Tsakris. 1992. Imipenem resistance in Enterobacter aerogenes is associated with derepression of chromosomal cephalosporinases and impaired permeability. FEMS Microbiol. Lett. 74: 195-199. PMID: 1526451

11. Chollet R, Bollet C, Chevalier J, Mallea M, Pages JM, Davin-Regli A. mar operon involved in multidrug resistance of Enterobacter aerogenes. Antimicrob Agents Chemother, 2002; 46: 10931097. PMID: 11897595

12. Alekshun, M.N., Y.S. Kim, and S.B. Levy. 2000. Mutational analysis of MarR, the negative regulator of marRAB expression in Escherichia coli, suggests the presence of two regions required for DNA binding. Mol. Microbiol. 35: 1394-1404. PMID: 10760140.

13. Oethinger, M., L. Podglajen, W.V. Kern, and S.B. Levy. 1998. Overexpression of the marA or soxS regulatory gene in clinical topoisomerase mutants of Escherichia coli. Antimicrob. Agents Chemother. 42: 2089-2094. PMID: 9687412.

14. 14- Cohen, S.P., Y. William, and S.B. Levy. 1993. A multidrug resistance regulatory chromosomal locus is widespread among enteric bacteria. J. Infect. Dis. 168: 484-488. PMID: 8335992

15. Gregory Bisson, MD; Neil O. Fishman, MD; Jean Baldus Patel, PhD; Paul H. Edelstein, MD; Ebbing Lautenbach, MD, MPH. Extended-Spectrum B-Lactamase-Producing Escherichia coli and Klebsiella Species: Risk Factors for Colonization and Impact of Antimicrobial Formulary Interventions on Colonization Prevalence Infection Control and Hospital, Epidemiology, Vol. 23, No. 5 (May 2002) (pp. 254-260)

16. CDC: Action needed now to halt spread of deadly bacteria: Data show more inpatients suffering infections from bacteria resistant to all or nearly all antibiotics". The Centers for Disease Control. March 5, 2013

17. Lowe, C; Katz, K; McGeer, A; Muller, MP; Toronto Esbl Working, Group (2012). "Disparity in infection control practices for multidrug-resistant Enterobacteriaceae". American journal of infection control, 40 (9): 836-9. PMID: 22361360
18. Lowe CF, Katz K, McGeer AJ, Muller MP, for the Toronto ESBL Working Group Efficacy of admission screening for extendedspectrum B-lactamase producing Enterobacteriaceae. PLoS One. 2013;8: e62678. doi: 10.1371/journal.pone.0062678. PMID: 23638132

19. Seoane AS, Levy SB. Characterization of MarR, the repressor of the multiple antibiotic resistance (mar) operon in Escherichia coli. J Bacteriol. 1995; 177(12): 3414-3419. PMID: 7768850

20. Cohen SP, Hachler H, Levy SB (1993) Genetic and functional analysis of the multiple antibiotic resistance (mar) locus in Escherichia coli. J Bacteriol 175: 1484-1492. PMID: 8383113.

\section{Comment on this article:}
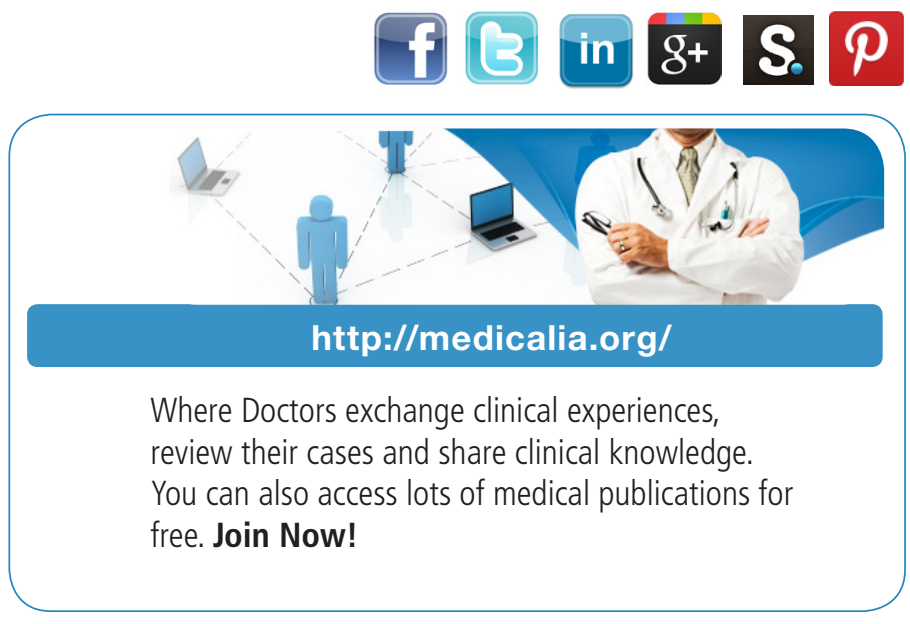

\section{Publish with iMedPub}

\section{http://www.imed.pub}

International Archives of Medicine is an open access journal publishing articles encompassing all aspects of medical science and clinical practice. IAM is considered a megajournal with independent sections on all areas of medicine. IAM is a really international journal with authors and board members from all around the world. The journal is widely indexed and classified Q1 in category Medicine. 